DR. FOAD TAGHDIRI (Orcid ID : 0000-0002-3280-2804)

Article type : Original Article

\title{
Neurofilament-Light in Former Athletes: A Potential Biomarker of Neurodegeneration and Progression
}

\section{Running title: Neurofilament Light in Concussion}

Foad Taghdiri, MD, MSc ${ }^{1}$, Namita Multani, $\mathrm{MSc}^{1}$, Miracle Ozzoude, $\mathrm{BSc}^{1}$, Apameh Tarazi, $\mathrm{MD}^{2,3}$, Mozhgan Khodadadi, $\mathrm{MA}^{3}$, Richard Wennberg, $\mathrm{MD}^{2,3,4}$, David Mikulis, $\mathrm{MD}^{3,4,5}$, Robin Green, $\mathrm{PhD}^{3,6}$, Brenda Colella,MSc ${ }^{3,6}$, Karen Deborah Davis, $\mathrm{PhD}^{3,4,7,8}$, Kaj Blennow,MD ${ }^{9,10}$, Henrik Zetterberg, MD $9,10,11,12$, Charles Tator, $\mathrm{MD}, \mathrm{PhD}^{3,13}$ and Maria Carmela Tartaglia, $\mathrm{MD}^{1,2,3,4,{ }^{*}}$

${ }^{1}$ Tanz Centre for Research in Neurodegenerative Diseases, University of Toronto, 60 Leonard avenue, Toronto, Canada

${ }^{2}$ Division of Neurology, Krembil Neuroscience Centre, 399 Bathurst St., Toronto, Canada

${ }^{3}$ Canadian Concussion Centre, Toronto Western Hospital, Krembil Brain Institute, University Health Network, 399 Bathurst St., Toronto, Canada.

${ }^{4}$ Institute of Medical Science, University of Toronto, 1 King's College Circle, Toronto, Canada ${ }^{5}$ Division of Neuroradiology, Joint Department of Medical Imaging, University Health Network, 399 Bathurst St., Toronto, Canada

${ }^{6}$ Department of Rehabilitation Sciences, University of Toronto, 500 University Ave, Toronto, Canada

${ }^{7}$ Department of Surgery, University of Toronto, 149 College St., Toronto, Canada

${ }^{8}$ Division of Brain, Imaging and Behaviour-systems Neuroscience, Krembil Brain Institute, University Health Network, 399 Bathurst St., Toronto, Canada

This article has been accepted for publication and undergone full peer review but has not been through the copyediting, typesetting, pagination and proofreading process, which may lead to differences between this version and the Version of Record. Please cite this article as doi: 10.1111/ENE.14251

This article is protected by copyright. All rights reserved 
${ }^{9}$ Institute of Neuroscience and Physiology, Department of Psychiatry and Neurochemistry, The Sahlgrenska Academy at the University of Gothenburg, Mölndal, Su Sahlgrenska, 41345 Göteborg, Sweden;

${ }^{10}$ Clinical Neurochemistry Laboratory, Sahlgrenska University Hospital, Mölndal, 43180 Sweden;

${ }^{11}$ Department of Neurodegenerative Disease, UCL Institute of Neurology, Queen Square, London, WC1N 3BG UK;

${ }^{12}$ UK Dementia Research Institute at UCL, University College London, London, WC1N 3BG UK

${ }^{13}$ Division of Neurosurgery, Toronto Western Hospital, Krembil Brain Institute, University Health Network, 399 Bathurst St., Toronto, Canada

\section{*Corresponding-author:}

\section{Dr. Maria Carmela Tartaglia MD, FRCPC,}

Associate Professor, University of Toronto

Tanz Centre for Research in Neurodegenerative Diseases,

Krembil Discovery Tower,

60 Leonard avenue, $6^{\text {th }}$ floor $6 \mathrm{KD}-407$,

Toronto, ON M5T 2S8, Canada

Tel: 416603 5483; Fax: 4166035768

Email: carmela.tartaglia@uhn.ca

This article is protected by copyright. All rights reserved 


\section{Abstract}

Background/Objective: This study aimed to evaluate serum neurofilament light chain (NF-L) levels in former professional contact sports athletes with multiple concussions (ExPro) as a potential biomarker of neurodegeneration and predictor of white-matter (WM) abnormality progression. Methods: Concentrations of NF-L in the serum of fifty-two cognitively normal ExPro and twentyone healthy controls ( $\mathrm{HC}$ ) with no history of concussions were measured using single molecule array (Simoa) technology. Both groups underwent neuroimaging at the time of serum collection. Eighteen of the participants in the ExPro underwent follow-up imaging after 2 years.

Results: Levels of serum NF-L were not significantly different between the ExPro and HC. However, in the ExPro group, NF-L levels were positively correlated with the mean diffusivity (MD) of corpus callosum (CC) and fornix, and total ventricular volume. Moreover, NF-L levels in the ExPro group at the first visit were positively correlated with the amount of increase in CC MD at the 2-year followup.

Conclusions: NF-L levels reflect neuronal changes in the ExPro group and predict the extent of decrease in white matter integrity over time. Serum NF-L might be a biomarker of neurodegeneration and WM abnormality progression in ExPro.

Keywords: Concussion; repetitive-concussions; Chronic-traumatic-encephalopathy; Biomarker; Diffusion tensor imaging;

This article is protected by copyright. All rights reserved 


\section{Introduction:}

Developing late-life cognitive impairment or neurodegeneration after repetitive concussions or sub-concussive impacts is a growing concern $(1,2)$. However, a reliable and easy-to-obtain biomarker to aid diagnosis and monitor neurodegeneration over time is still lacking. The ease of venipuncture makes blood biomarkers attractive for this purpose. Neurofilament light protein (NF-L) is among the most promising biomarker candidates(3). Neurofilaments are major components of the axonal cytoskeleton and consist of three types of protein chains: a light chain, an intermediate chain, and a heavy chain(4). In neurodegenerative diseases, axonal damage may lead to release of the neurofilaments into the CSF and blood(5,6).

Several studies have reported increased concentrations of serum NF-L in individuals with neurodegenerative diseases(3,7-9). In addition, recent studies have suggested that serum NF-L may be a sensitive biomarker of concussion $(10,11)$. Increased levels of serum NF-L have been reported in amateur boxers after a bout with higher levels in boxers who had received more head blows(11). Importantly, NF-L levels decreased after three months of rest from boxing, but were still higher than in controls(11). Similarly, levels of NF-L were observed to increase over the course of a season in American football players(10), suggesting that NF-L could be a marker of concussion or repeated subconcussive head impacts. However, the utility of serum NF-L as a biomarker of neurodegeneration in patients with a history of remote concussions has not been studied.

Neuroimaging techniques are widely used to investigate brain changes in contact sports athletes. The most frequently reported white-matter (WM) abnormalities observed in sport-related concussions are in the corpus-callosum (CC), uncinate-fasciculus (UNC), superior longitudinal fasciculus $(\mathrm{SLF})$, cingulum $(\mathrm{Cg})$, and fornix $(\mathrm{Fx})(12,13)$. Alterations in the brain resting state networks, particularly in the default mode network (DMN), have also been observed in several sportrelated concussion studies(14). Moreover, greater brain atrophy such as greater hippocampal atrophy and ventricular size than expected for age has been reported in former athletes with multiple concussions compared to healthy controls(15).

The aims of this study were: Aim 1. To compare serum NF-L levels, WM integrity, subcortical volumes, and DMN functional connectivity (FC) between former professional contact 
sports athletes with a history of multiple concussions (ExPro), and healthy controls (HC). Aim 2. To assess the relationship between the serum NF-L levels and concussion history, performance on neuropsychiatric assessments, WM integrity, subcortical volumes, and DMN connectivity. Aim 3. To assess whether serum NF-L can predict the longitudinal changes in WM integrity, subcortical volumes, and DMN connectivity in a subgroup of ExPro who have returned for 2-year follow up.

\section{Methods:}

\section{Participants:}

A total of seventy-three participants participated in this study: (a) ExPro group: fifty-two cognitively normal former professional contact sports players ( 2 women, 50 men, age [mean \pm SD] $54.5 \pm 12.9$ years) with a history of multiple concussions were recruited through the Canadian Concussion Centre, University Health Network (UHN), Toronto, Canada. Concussion history (e.g., number of concussions, date of last concussion, date of first concussion, and number of years played) was based on participants' self-report. Key exclusion criteria were the inability to recall the number of concussions, history of neurological or severe psychiatric disorder, or other illness affecting the brain, developmental disorder, or any lesions on routine magnetic resonance imaging (MRI); (b) HC group: twenty-one participants (all men, age [mean \pm SD] $49.4 \pm 10.1$ years). Healthy controls were recruited from the community and had no history of concussions, neurological or psychiatric disease. All participants underwent blood tests, neuroimaging, and neuropsychological assessments. Eighteen ExPro participants (all male, age[mean $\pm \mathrm{SD}] 55.4 \pm 13.1$ years) underwent a 2-year follow-up assessment. The research ethics boards of the University Health Network approved the study. Consent was obtained from all participants before participating in the study.

The details of methods used for this study can be found in Supplementary-file 1.

Data Availability Statement: All data can be shared at the request of other investigators. 


\section{Results}

Table-1a summarizes participants' demographic information. Serum NF-L levels significantly correlated with age of the participants $(\mathrm{r}=0.626, \mathrm{p}<0.0001)$. However, no significant correlations were found between the levels of NF-L and number of reported concussions, interval from last concussion, and number of years of professional play $(\mathrm{r}=0.28$, unadjusted $\mathrm{p}=0.1 ; \mathrm{r}=0.075$, unadjusted $\mathrm{p}=0.67$; and $\mathrm{r}=0.097$, unadjusted $\mathrm{p}=0.581$; respectively) in the ExPro group.

Serum NF-L difference between ExPro and HC groups

No significant between-subjects (i.e., ExPro and HC groups) effects were found on the serum NF-L levels $(\mathrm{F}=0.09, \mathrm{p}=0.763)$.

\section{Effects of ExPro and HC groups on CC integrity and volume}

After Bonferroni correction for multiple comparisons, the only WM tract integrity which was significantly affected by the ExPro and $\mathrm{HC}$ groups was that of the $\mathrm{CC}$ as measured by the MD and $\mathrm{RD}$ of this tract $(\mathrm{F}=10.6, \mathrm{p}=0.016 ; \mathrm{F}=12.7, \mathrm{p}=0.008$; respectively) (Table-1b).

The CC volume was also significantly smaller in the ExPro group than the HC group $\left(1.8 \pm 0.3 \times 10^{-3}\right.$ vs $2.0 \pm 0.3 \times 10^{-3}$, between-subjects $\mathrm{p}=0.026$ ).

\section{Effects of ExPro and HC groups on Resting state dorsal DMN}

Between group FC maps within the DMN showed significant increase in FC between the right hippocampus and the right superior frontal gyrus (SFG) in the ExPro group compared to HC (T=3.32, controlled for age, unadjusted $\mathrm{p}=0.001, \mathrm{FDR}-\mathrm{p}=0.01)$ (Figure-1). We did not find any statistically significant effects of the ExPro and $\mathrm{HC}$ in $\mathrm{FC}$ on the other DMN components.

\section{Effects of ExPro and HC groups on Hippocampi and total ventricular volumes}

After controlling for age and Bonferroni corrections, no significant effect of group were found on the right hippocampus $(\mathrm{F}=1.45, \mathrm{p}=0.7)$, left hippocampus $(\mathrm{F}=0.2, \mathrm{p}=1.0)$, or total ventricular $(\mathrm{F}=3.15$, $\mathrm{p}=0.28$ ) volumes.

This article is protected by copyright. All rights reserved 
Relationship between serum NF-L levels and brain measures which were found to be different between the groups

Serum NF-L levels were significantly correlated (controlled for age) with the MD and RD of the CC in the ExPro group ( $r=0.315, p=0.024 ; r=0.303, p=0.031$; respectively) but not in the $\mathrm{HC}$ group ( $r=-$ $0.017, p=0.942 ; r=-0.122, p=0.640$; respectively). However, a trend towards significance was noted in correlation between serum NF-L levels and the CC volume after controlling for age in the ExPro group $(\mathrm{r}=-0.264, \mathrm{p}=0.067)$. The correlation between the two was not significant in the HC group $(\mathrm{r}=-$ $0.073, \mathrm{p}=0.767)$.

No significant correlations were found between the NF-L levels and FC between the right SFG and hippocampus in ExPro and $\mathrm{HC}$ groups ( $\mathrm{r}=-0.130, \mathrm{p}=0.364 ; \mathrm{r}=0.087, \mathrm{p}=0.717$; respectively).

\section{Post-hoc correlation analyses}

In post-hoc analyses, we also examined correlations in the ExPro group between the serum NF-L and other brain measures that showed no statistical significant difference between the ExPro and $\mathrm{HC}$ groups. These results are summarized in Table-2. After correcting for multiple comparisons, NF-L levels were significantly correlated only with the integrity of the Fx and total ventricular volume (Table-2).

In the dorsal DMN, after correcting for age, the main effect of NF-L on FC between the right and left hippocampi was significant ( $\mathrm{T}=-2.93$, unadjusted $\mathrm{p}=0.005$, FDR- $\mathrm{p}=0.04$ ).

Relationship between levels of serum NF-L and neuropsychological assessments in ExPro group The serum NF-L was negatively correlated with the memory and speed of processing composite scores $(\mathrm{r}=-0.349, \mathrm{p}=0.014 ; \mathrm{r}=-0.289, \mathrm{p}=0.042$; respectively). However, no significant correlation was found with the executive functioning composite score $(\mathrm{r}=0.044, \mathrm{p}=0.759)$ (Table-3).

\section{Longitudinal follow-up of eighteen participants in the ExPro group}

In addition to the significant cross-sectional correlation between the serum NF-L levels and CC MD in the ExPro group when investigated cross-sectionally, the levels of NF-L at the first visit were also found to be significantly correlated with the change of $\mathrm{MD}, \mathrm{RD}$, and FA of the $\mathrm{CC}$ over time in the

This article is protected by copyright. All rights reserved 
subgroup of ExPro participants who returned for their 2-year follow-up ( $r=0.567, p=0.018 ; r=0.627$, $\mathrm{p}=0.007$; and $\mathrm{r}=-0.571, \mathrm{p}=0.017$; respectively) (Figure-2). However, serum NF-L levels were not significantly correlated with the change in the $\mathrm{CC}$ AD and $\mathrm{CC}$ volume over the 2-year period $(\mathrm{r}=0.370, \mathrm{p}=0.144$; and $\mathrm{r}=-0.168, \mathrm{p}=0.519$; respectively $)$.

In post-hoc analyses, no significant correlations were found between the NF-L and longitudinal change over the 2 years in $\mathrm{MD}, \mathrm{AD}, \mathrm{RD}$, and FA of the $\mathrm{Fx}(\mathrm{r}=0.134$, unadjusted $\mathrm{p}=0.608$; $\mathrm{r}=0.096$, unadjusted $\mathrm{p}=0.715 ; \mathrm{r}=0.149$, unadjusted $\mathrm{p}=0.567 ; \mathrm{r}=-0.076$, unadjusted $\mathrm{p}=0.772$; respectively) or between NF-L and change in total ventricular volume over a 2-year period $(r=0.267$, unadjusted $\mathrm{p}=0.301)$. Furthermore, no significant effect of baseline serum NF-L was found on longitudinal changes in connectivity of the right hippocampus and SFG (unadjusted $\mathrm{p}=0.795$ ) and connectivity of the right and left hippocampi (unadjusted- $p=0.711$ ) after 2 years.

\section{Discussion:}

Results of this study support that the serum levels of NF-L reflect cerebral abnormalities in the ExPro group. Our findings revealed that the ExPro and HC groups showed significantly different integrity and volume of the CC and that serum NF-L was correlated with these measures in the ExPro group. In addition, we found that NF-L was associated with integrity of the Fx, total ventricular volume, and resting-state FC of the right and left hippocampi in the ExPro group. Remarkably, the level of serum NF-L at the first visit was predictive of the decrease in integrity of the CC in the subgroup of ExPro participants who had a 2-year follow-up.

Although, we did not find any significant difference in the NF-L levels between the ExPro and HC groups, many studies have suggested that NF-L should be considered as a signature of neurodegeneration(6). A higher risk of neurodegeneration has been reported in some studies in athletes who suffered multiple concussions compared to the general population(2). Thus, levels of NF-L might be expected to be elevated in contact sports athletes with a history of multiple concussions, however, it should be noted that many athletes never develop neurodegenerative diseases over the course of their lives(16). Thus, group comparisons of serum NF-L between former athletes with multiple concussions and controls may not show differences. Nonetheless, NF-L may still be a useful biomarker that can reflect cerebral structural and functional changes in these population. 
Abnormal CC integrity in patients with a history of concussion is a common finding in the literature(13). Similarly, reduced CC integrity has also been reported in retired asymptomatic contact sports athletes with a history of multiple concussions(17). It has been suggested that concussion affects the brain synergistically with the normal aging process and consequently leads to structural and functional deficits which are beyond the normal aging process alone(15). WM fibers that constitute the CC are highly susceptible to the effects of both concussion(13) and aging(18). Breakdown of these fibers may cause NF-L to be released and detected in CSF or blood(19). In our study, the significant correlations between NF-L levels and different brain structures' integrity, volume, or FC (e.g., integrity of the $\mathrm{CC}$ and Fx, CC volume, total ventricular volume, and right and left hippocampi connectivity) after controlling for the effects of age indicated neurodegeneration that is beyond the normal aging process alone in the ExPro participants. In addition, since levels of NF-L were only associated with the change in WM integrity of the $\mathrm{CC}$, in the subgroup of the ExPro participants who were followed up longitudinally, this suggests that loss of CC integrity is the earliest neurodegenerative change over the relatively short period of 2 years. This longitudinal observation highlights the potential prognostic power of serum NF-L levels in patients with multiple concussions.

In the present study, we observed increased FC between the right SFG and hippocampus in the ExPro group compared to the HC group. The hippocampus-frontal lobe circuit has been widely investigated in stress and depression in human and animal models(20,21). Given that stress is a commonly reported symptom in former athletes with multiple concussions(22), the observed increased resting-state co-activation of the SFG and hippocampus might be attributed to the elevated levels of glutamate in these structures in the ExPro group compared to HC.

The findings of this study also showed that serum NF-L level offers a rapid and accessible measure of global atrophy of the brain as it was significantly correlated with the normalized total ventricular volume in the ExPro group. These findings are consistent with the studies that looked at similar correlations in other neurodegenerative diseases such as $\mathrm{AD}(23)$, and $\operatorname{HD}(24)$. Furthermore, NF-L levels correlated with the integrity of the Fx and the resting state connectivity of the right and left hippocampi in the ExPro group. The hippocampus is involved in memory function(25), speed of processing(26), and executive function(27), processes often affected in those with multiple concussions. The Fx is the major linking pathway between the hippocampus and other brain 
regions(28). Some studies have confirmed that the right and left hippocampi are functionally and structurally connected $(29,30)$. Thus, alterations in the interhemispheric communication between the right and left hippocampi may impair cognition(31).

While the current study showed promising results in terms of using serum NF-L as a potential biomarker for neurodegeneration in former professional athletes with a history of multiple concussions, potential limitations should be noted. The small sample size in the longitudinally studied subgroup as well as the small number of female participants in this study prevents determination of the generalizability of our results. In addition, other comorbidities such as hypertension, obesity, or sleep apnea which were not considered in this study may play important roles in the Therefore, more studies with a larger sample size including both men and women are required to confirm and extend the results of this study. Also, it should be noted that through our study we cannot determine causation. Although, repetitive concussions may explain our findings, comorbidities such as hypertension(32), obesity(33), or sleep apnea(34) should be considered as potential confounding factors as they may be responsible for imaging differences which are seen between individuals. In addition, we followed up the ExPro subgroup for only 2 years which is a relatively short period to track neurodegeneration. In addition, in our study we did not follow up HC subgroup. Longer followup periods of both Expro and HC groups are necessary to accurately assess the use of NF-L to predict longitudinal structural and functional brain changes.

In conclusion, our findings indicate that the measurement of serum NF-L levels is a potentially promising biomarker of neurodegeneration as well as a prognostic biomarker of progression in ExPro. However, based solely on these findings, and before investigating the brain pathology of the participants, no conclusion can be made on whether NF-L levels can serve as a diagnostic biomarker of chronic traumatic encephalopathy.

\section{Acknowledgements:}

We'd like to thank all participants for generous donation of their time to participate in this research program. We'd like to thank Leo Ezerins, director of CFLAA for his help with this study. We thank 
E. Hlasny, K. Ta, B. Li and Dr. A. Crawley for their support in image acquisition. HZ is a Wallenberg Academy Fellow supported by grants from the Swedish Research Council (\#2018-02532), the European Research Council (\#681712) and Swedish State Support for Clinical Research (\#ALFGBG720931).

This article is protected by copyright. All rights reserved 


\section{Reference:}

1. Abner EL, Nelson PT, Schmitt FA, Browning SR, Fardo DW, Wan L, et al. Self-reported head injury and risk of late-life impairment and AD pathology in an AD center cohort. Dementia and geriatric cognitive disorders. 2014;37(5-6):294-306.

2. McKee AC, Cantu RC, Nowinski CJ, Hedley-Whyte ET, Gavett BE, Budson AE, et al. Chronic traumatic encephalopathy in athletes: progressive tauopathy after repetitive head injury. Journal of Neuropathology \& Experimental Neurology. 2009;68(7):709-35.

3. Gaiottino J, Norgren N, Dobson R, Topping J, Nissim A, Malaspina A, et al. Increased neurofilament light chain blood levels in neurodegenerative neurological diseases. PloS one. 2013;8(9):e75091.

4. Petzold A. Neurofilament phosphoforms: surrogate markers for axonal injury, degeneration and loss. Journal of the neurological sciences. 2005;233(1-2):183-98.

5. Gisslén M, Price RW, Andreasson U, Norgren N, Nilsson S, Hagberg L, et al. Plasma concentration of the neurofilament light protein (NFL) is a biomarker of CNS injury in HIV infection: a crosssectional study. EBioMedicine. 2016;3:135-40.

This article is protected by copyright. All rights reserved 
6. Lewczuk P, Ermann N, Andreasson U, Schultheis C, Podhorna J, Spitzer P, et al. Plasma neurofilament light as a potential biomarker of neurodegeneration in Alzheimer's disease. Alzheimer's research \& therapy. 2018;10(1):71.

7. Weston PS, Poole T, Ryan NS, Nair A, Liang Y, Macpherson K, et al. Serum neurofilament light in familial Alzheimer disease: a marker of early neurodegeneration. Neurology. 2017;89(21):2167-75.

8. Byrne LM, Rodrigues FB, Blennow K, Durr A, Leavitt BR, Roos RA, et al. Neurofilament light protein in blood as a potential biomarker of neurodegeneration in Huntington's disease: a retrospective cohort analysis. The Lancet Neurology. 2017;16(8):601-9.

9. Ashton NJ, Leuzy A, Lim YM, Troakes C, Hortobágyi T, Höglund K, et al. Increased plasma neurofilament light chain concentration correlates with severity of post-mortem neurofibrillary tangle pathology and neurodegeneration. Acta neuropathologica communications. 2019;7(1):1-11.

10. Oliver JM, Jones MT, Kirk KM, Gable DA, Repshas JT, Johnson TA, et al. Serum neurofilament light in American football athletes over the course of a season. Journal of neurotrauma. 2016;33(19):1784-9.

11. Shahim P, Zetterberg H, Tegner $Y$, Blennow K. Serum neurofilament light as a biomarker for mild traumatic brain injury in contact sports. Neurology. 2017;10-1212.

12. Taghdiri F, Varriano B, Tartaglia MC. Assessment of Oculomotor Function in Patients With Postconcussion Syndrome: A Systematic Review. The Journal of Head Trauma Rehabilitation. 2017;

13. Khong E, Odenwald N, Hashim E, Cusimano MD. Diffusion tensor imaging findings in postconcussion syndrome patients after mild traumatic brain injury: a systematic review. Frontiers in neurology. 2016;7.

This article is protected by copyright. All rights reserved 
14. Abbas K, Shenk TE, Poole VN, Breedlove EL, Leverenz LJ, Nauman EA, et al. Alteration of default mode network in high school football athletes due to repetitive subconcussive mild traumatic brain injury: a resting-state functional magnetic resonance imaging study. Brain connectivity. 2015;5(2):91-101.

15. Misquitta K, Dadar M, Tarazi A, Hussain MW, Alatwi MK, Ebraheem A, et al. The relationship between brain atrophy and cognitive-behavioural symptoms in retired Canadian football players with multiple concussions. Neurolmage: Clinical. 2018;19:551-8.

16. Hazrati L-N, Tartaglia MC, Diamandis P, Davis K, Green RE, Wennberg R, et al. Absence of chronic traumatic encephalopathy in retired football players with multiple concussions and neurological symptomatology. Frontiers in human neuroscience. 2013;7:222.

17. Tremblay S, Henry LC, Bedetti C, Larson-Dupuis C, Gagnon J-F, Evans AC, et al. Diffuse white matter tract abnormalities in clinically normal ageing retired athletes with a history of sportsrelated concussions. Brain. 2014;137(11):2997-3011.

18. Ota $\mathrm{M}$, Obata $\mathrm{T}$, Akine $\mathrm{Y}$, Ito $\mathrm{H}$, Ikehira $\mathrm{H}$, Asada $\mathrm{T}$, et al. Age-related degeneration of corpus callosum measured with diffusion tensor imaging. Neuroimage. 2006;31(4):1445-52.

19. Zetterberg H, Smith DH, Blennow K. Biomarkers of mild traumatic brain injury in cerebrospinal fluid and blood. Nature Reviews Neurology. 2013;9(4):201.

20. McEwen BS, Nasca C, Gray JD. Stress effects on neuronal structure: hippocampus, amygdala, and prefrontal cortex. Neuropsychopharmacology. 2016;41(1):3.

21. Bagley J, Moghaddam B. Temporal dynamics of glutamate efflux in the prefrontal cortex and in the hippocampus following repeated stress: effects of pretreatment with saline or diazepam. Neuroscience. 1997;77(1):65-73.

This article is protected by copyright. All rights reserved 
22. Gouttebarge V, Aoki H, Lambert M, Stewart W, Kerkhoffs G. A history of concussions is associated with symptoms of common mental disorders in former male professional athletes across a range of sports. The Physician and sportsmedicine. 2017;45(4):443-9.

23. Mattsson N, Andreasson U, Zetterberg H, Blennow K. Association of plasma neurofilament light with neurodegeneration in patients with Alzheimer disease. JAMA neurology. 2017;74(5):55766.

24. Byrne LM, Rodrigues FB, Blennow K, Durr A, Leavitt BR, Roos RA, et al. Neurofilament light protein in blood as a potential biomarker of neurodegeneration in Huntington's disease: a retrospective cohort analysis. The Lancet Neurology. 2017;16(8):601-9.

25. Olton DS, Becker JT, Handelmann GE. Hippocampus, space, and memory. Behavioral and Brain Sciences. 1979;2(3):313-22.

26. Papp KV, Kaplan RF, Springate B, Moscufo N, Wakefield DB, Guttmann CR, et al. Processing speed in normal aging: Effects of white matter hyperintensities and hippocampal volume loss. Aging, Neuropsychology, and Cognition. 2014;21(2):197-213.

27. Reuben A, Brickman AM, Muraskin J, Steffener J, Stern Y. Hippocampal atrophy relates to fluid intelligence decline in the elderly. Journal of the International Neuropsychological Society. 2011;17(1):56-61.

28. Duvernoy HM. The human hippocampus: an atlas of applied anatomy. JF Bergmann-Verlag; 2013.

29. Lacuey N, Zonjy B, Kahriman ES, Kaffashi F, Miller J, Lüders HO. Functional connectivity between right and left mesial temporal structures. Brain Structure and Function. 2015;220(5):2617-23.

30. Gloor P, Salanova V, Olivier A, Quesney L. The human dorsal hippocampal commissure: An anatomically identifiable and functional pathway. Brain. 1993;116(5):1249-73.

This article is protected by copyright. All rights reserved 
31. Zhou Y, Shu N, Liu Y, Song M, Hao Y, Liu H, et al. Altered resting-state functional connectivity and anatomical connectivity of hippocampus in schizophrenia. Schizophrenia research. 2008;100(13):120-32.

32. Sabisz A, Naumczyk P, Marcinkowska A, Graff B, Gąsecki D, Glinska A, et al. Aging and hypertension-independent or intertwined white matter impairing factors? Insights from the quantitative Diffusion Tensor Imaging. Frontiers in aging neuroscience. 2019;11:35.

33. Xu J, Li Y, Lin H, Sinha R, Potenza MN. Body mass index correlates negatively with white matter integrity in the fornix and corpus callosum: a diffusion tensor imaging study. Human brain mapping. 2013;34(5):1044-52.

34. Chen H-L, Huang C-C, Lin H-C, Lu C-H, Chen P-C, Chou K-H, et al. White matter alteration and autonomic impairment in obstructive sleep apnea. Journal of Clinical Sleep Medicine. 2020;16(2):293-302.

This article is protected by copyright. All rights reserved 
Figure-1 Increased resting-state functional connectivity between the right superior frontal gyrus (SFG) and right hippocampus in the ExPro group compared to the HC group.

Figure-2 Correlation between the serum NF-L levels at first visit and a) the change in CC MD (presented as $\mathrm{mm}^{2} \cdot \mathrm{s}^{-1} \cdot 10^{-3}$ ) after 2-year follow-up b) CC MD (presented as $\mathrm{mm}^{2} \cdot \mathrm{s}^{-1} \cdot 10^{-3}$ ) at first visit and after 2-year follow-up

Supplementary File 1 Details of neuroimaging processes

This article is protected by copyright. All rights reserved 
Table-1 Comparing the ExPro and HC groups

a) Participants characteristics

\begin{tabular}{|c|c|c|c|c|c|c|c|c|}
\hline \multirow{3}{*}{ ExPro group } & $\mathbf{N}$ & Age (years) & $\begin{array}{c}\text { Gender } \\
\text { (female/mal } \\
\text { e) }\end{array}$ & $\begin{array}{c}\text { Years of } \\
\text { education }\end{array}$ & Sports played & $\begin{array}{c}\text { Number of reported } \\
\text { concussions }\end{array}$ & $\begin{array}{c}\text { Interval between last } \\
\text { concussion and } \\
\text { testing (years) }\end{array}$ & $\begin{array}{c}\text { Number of years } \\
\text { played } \\
\text { professionally }\end{array}$ \\
\hline & & & & & Football:43; & & $20.47 \pm 16.0$ & \\
\hline & 52 & $54.5 \pm 12.9$ & $2 / 50$ & $15.8 \pm 1.84$ & $\begin{array}{l}\text { Hockey:8; } \\
\text { Boxing:1 }\end{array}$ & $5.75 \pm 5.1$ (median:4) & $($ median:21.0) & $7.63 \pm 3.9$ \\
\hline HC group & 21 & $49.4 \pm 10.1$ & $0 / 21$ & $15.9 \pm 1.81$ & N/A & 0 & N/A & 0 \\
\hline $\begin{array}{r}\text { Unadjusted } \\
\text { p value }\end{array}$ & - & 0.11 & 1.00 & 0.806 & - & - & - & - \\
\hline
\end{tabular}

b) Comparing DTI measures between the ExPro and HC groups

\begin{tabular}{|c|c|c|c|c|c|c|c|c|c|}
\hline & FA & $\mathrm{MD}^{\dagger}$ & $\mathrm{AD}^{\dagger}$ & $\mathrm{RD}^{\dagger}$ & & FA & $\mathrm{MD}^{\dagger}$ & $\mathrm{AD}^{*}$ & $\mathrm{RD}^{\dagger}$ \\
\hline Right SLF & & & & & Left SLF & & & & \\
\hline ExPro group & $0.383 \pm 0.03$ & $0.825 \pm 0.04$ & $1.17 \pm 0.04$ & $0.654 \pm 0.05$ & ExPro group & $0.391 \pm 0.02$ & $0.818 \pm 0.03$ & $1.16 \pm 0.04$ & $0.645 \pm 0.03$ \\
\hline HC group & $0.380 \pm 0.02$ & $0.816 \pm 0.03$ & $1.15 \pm 0.03$ & $0.650 \pm 0.03$ & $\mathrm{HC}$ group & $0.388 \pm 0.02$ & $0.800 \pm 0.03$ & $1.13 \pm 0.02$ & $0.630 \pm 0.03$ \\
\hline Unadjusted & 0.383 & 0.535 & 0.137 & 0.885 & Unadjusted & 0.400 & 0.061 & 0.006 & 0.219 \\
\hline $\mathrm{p}$ value & & & & & $\mathrm{p}$ value & & & & \\
\hline Right UNC & & & & & Left UNC & & & & \\
\hline ExPro group & $0.361 \pm 0.03$ & $0.913 \pm 0.05$ & $1.28 \pm 0.06$ & $0.728 \pm 0.06$ & ExPro group & $0.352 \pm 0.04$ & $0.906 \pm 0.07$ & $1.26 \pm 0.07$ & $0.730 \pm 0.07$ \\
\hline HC group & $0.346 \pm 0.04$ & $0.901 \pm 0.06$ & $1.24 \pm 0.07$ & $0.728 \pm 0.07$ & $\mathrm{HC}$ group & $0.330 \pm 0.04$ & $0.893 \pm 0.07$ & $1.21 \pm 0.06$ & $0.733 \pm 0.08$ \\
\hline Unadjusted & 0.067 & 0.512 & 0.028 & 0.841 & Unadjusted & 0.019 & 0.661 & 0.025 & 0.672 \\
\hline $\mathrm{p}$ value & & & & & $\mathrm{p}$ value & & & & \\
\hline Right Cg & & & & & Left Cg & & & & \\
\hline ExPro group & $0.349 \pm 0.03$ & $0.880 \pm 0.05$ & $1.21 \pm 0.05$ & $0.712 \pm 0.05$ & ExPro group & $0.348 \pm 0.03$ & $0.893 \pm 0.05$ & $1.23 \pm 0.06$ & $0.723 \pm 0.07$ \\
\hline HC group & $0.342 \pm 0.02$ & $0.880 \pm 0.04$ & $1.20 \pm 0.03$ & $0.717 \pm 0.03$ & HC group & $0.347 \pm 0.03$ & $0.888 \pm 0.03$ & $1.22 \pm 0.04$ & $0.719 \pm 0.05$ \\
\hline Unadjusted & 0.215 & 0.702 & 0.665 & 0.473 & Unadjusted & 0.674 & 0.927 & 0.690 & 0.955 \\
\hline $\mathrm{p}$ value & & & & & $\mathrm{p}$ value & & & & \\
\hline CC & & & & & $\mathbf{F x}$ & & & & \\
\hline ExPro group & $0.479 \pm 0.03$ & $0.920 \pm 0.06$ & $1.45 \pm 0.06$ & $0.657 \pm 0.06$ & ExPro group & $0.208 \pm 0.04$ & $1.95 \pm 0.3$ & $2.33 \pm 0.3$ & $1.76 \pm 0.4$ \\
\hline $\mathrm{HC}$ group & $0.486 \pm 0.02$ & $0.877 \pm 0.03$ & $1.42 \pm 0.03$ & $0.606 \pm 0.04$ & $\mathrm{HC}$ group & $0.215 \pm 0.02$ & $1.89 \pm 0.1$ & $2.29 \pm 0.1$ & $1.69 \pm 0.1$ \\
\hline Unadjusted p & 0.373 & $0.002 *$ & 0.079 & $0.001 *$ & Unadjusted $\mathrm{p}$ & 0.726 & 0.685 & 0.852 & 0.608 \\
\hline value & & & & & value & & & & \\
\hline
\end{tabular}

*significant at the level of $\mathrm{p}<0.006$ after Bonferroni correction for multiple comparisons (controlled for age); ${ }^{\dagger}$ missing for 6 participants as they could not remember

the last concussion date; ${ }^{\ddagger}$ data presented as $\mathrm{mm}^{2} \cdot \mathrm{s}^{-1} .10^{-3}$; SLF, Superior longitudinal fasciculus; UNC, Uncinate fasciculus; Cg, Cingulum fasciculus; CC, Corpus

Callosum; Fx, Fornix bundle; FA, Fractional anisotropy; MD, Mean diffusivity; AD, Axial diffusivity; RD, Radial diffusivity; all p-values in section (b) calculated with using a general linear modelling analysis with group (i.e., Expro and $\mathrm{HC}$ ) as a fixed factor and age as a covariate

This article is protected by copyright. All rights reserved 
Table-2 Partial correlation with the serum NF-L levels after controlling for age (Post-hoc analysis)

\begin{tabular}{|c|c|c|c|c|c|}
\hline & $\mathbf{r}^{\dagger}$ & p value (unadjusted $p$ ) & & $\mathbf{r}^{\dagger}$ & p value (unadjusted p) \\
\hline R SLF & & & R UNC & & \\
\hline FA & 0.087 & N.S. $(0.543)$ & FA & -0.202 & N.S. $(0.156)$ \\
\hline MD & 0.014 & N.S. (0.922) & MD & 0.251 & N.S. $(0.075)$ \\
\hline $\mathrm{AD}$ & 0.097 & N.S. $(0.500)$ & $\mathrm{AD}$ & 0.157 & N.S. (0.272) \\
\hline $\mathrm{RD}$ & -0.026 & N.S. $(0.858)$ & $\mathrm{RD}$ & 0.258 & N.S. (0.067) \\
\hline L SLF & & & L UNC & & \\
\hline FA & 0.056 & N.S. (0.695) & FA & -0.058 & N.S. (0.684) \\
\hline MD & 0.139 & N.S. (0.329) & MD & 0.126 & N.S. $(0.378)$ \\
\hline $\mathrm{AD}$ & 0.189 & N.S. $(0.185)$ & $\mathrm{AD}$ & 0.105 & N.S. $(0.464)$ \\
\hline $\mathrm{RD}$ & 0.098 & N.S. (0.494) & $\mathrm{RD}$ & 0.122 & N.S. (0.392) \\
\hline $\mathbf{F x}$ & & & R Cg & & \\
\hline FA & -0.424 & $0.02 *(0.002)$ & FA & -0.009 & N.S. (0.949) \\
\hline MD & 0.474 & $0.004 *(<0.001)$ & MD & 0.220 & N.S. (0.122) \\
\hline $\mathrm{AD}$ & 0.457 & $0.01 *(0.001)$ & $\mathrm{AD}$ & 0.239 & N.S. $(0.091)$ \\
\hline $\mathrm{RD}$ & 0.479 & $0.004 *(<0.001)$ & RD & 0.185 & N.S. (0.194) \\
\hline Volume- & & & $\mathbf{L ~ C g}$ & & \\
\hline to-ICV & & & FA & 0.104 & N.S. (0.466) \\
\hline R hippo. & -0.352 & $0.13(\mathbf{0 . 0 1 3})$ & MD & -0.061 & N.S. (0.671) \\
\hline L hippo. & -0.347 & $0.15(\mathbf{0 . 0 1 5})$ & $\mathrm{AD}$ & -0.019 & N.S. $(0.892)$ \\
\hline Ventricular & 0.446 & $0.01 *(0.001)$ & RD & -0.075 & N.S. (0.599) \\
\hline
\end{tabular}

This article is protected by copyright. All rights reserved 
Table-3 Pearson's correlation between the NF-L and neuropsychological assessments in the ExPro group

\begin{tabular}{|c|c|c|}
\hline & $\mathrm{r}$ & $\begin{array}{c}\text { unadjusted } \\
\text { p value }\end{array}$ \\
\hline \multicolumn{3}{|l|}{ Memory Assessment } \\
\hline RAVLT trials 1-5 z-score & -0.284 & 0.043 \\
\hline RAVLT long-delay recall z-score & -0.254 & 0.072 \\
\hline RVDLT trials 1-5 z-score & -0.365 & 0.009 \\
\hline Memory composite score & -0.349 & $0.014 *$ \\
\hline \multicolumn{3}{|l|}{ Speed of Processing Assessment } \\
\hline SCNT z-score & -0.489 & $<0.001$ \\
\hline SWRT z-score & -0.328 & 0.018 \\
\hline SDMT written z-score & -0.244 & 0.084 \\
\hline SDMT oral z-score & -0.275 & 0.051 \\
\hline Speed of processing composite score & -0.289 & $0.042 *$ \\
\hline \multicolumn{3}{|l|}{ Executing Functioning Assessment } \\
\hline TMT part B z-score & -0.109 & 0.442 \\
\hline WCST percent error z-score & 0.043 & 0.767 \\
\hline WCST percent perseverative error z-score & 0.198 & 0.169 \\
\hline WCST percent non-perseverative error z-score & -0.247 & 0.081 \\
\hline WCST conceptual z-score & 0.021 & 0.882 \\
\hline Executive functioning composite score & 0.044 & 0.759 \\
\hline
\end{tabular}

*Composite scores significant at level of $\mathrm{p}<0.05$; RAVLT, Rey Auditory Verbal

Learning Test, RVDLT, Rey Visual Design Learning Test; SCNT, Stroop colour naming test; SWRT, Stroop word reading test; SDMT, Symbol digit modalities test; TMT, Trail making test; WCST, Wisconsin card sorting test

This article is protected by copyright. All rights reserved 


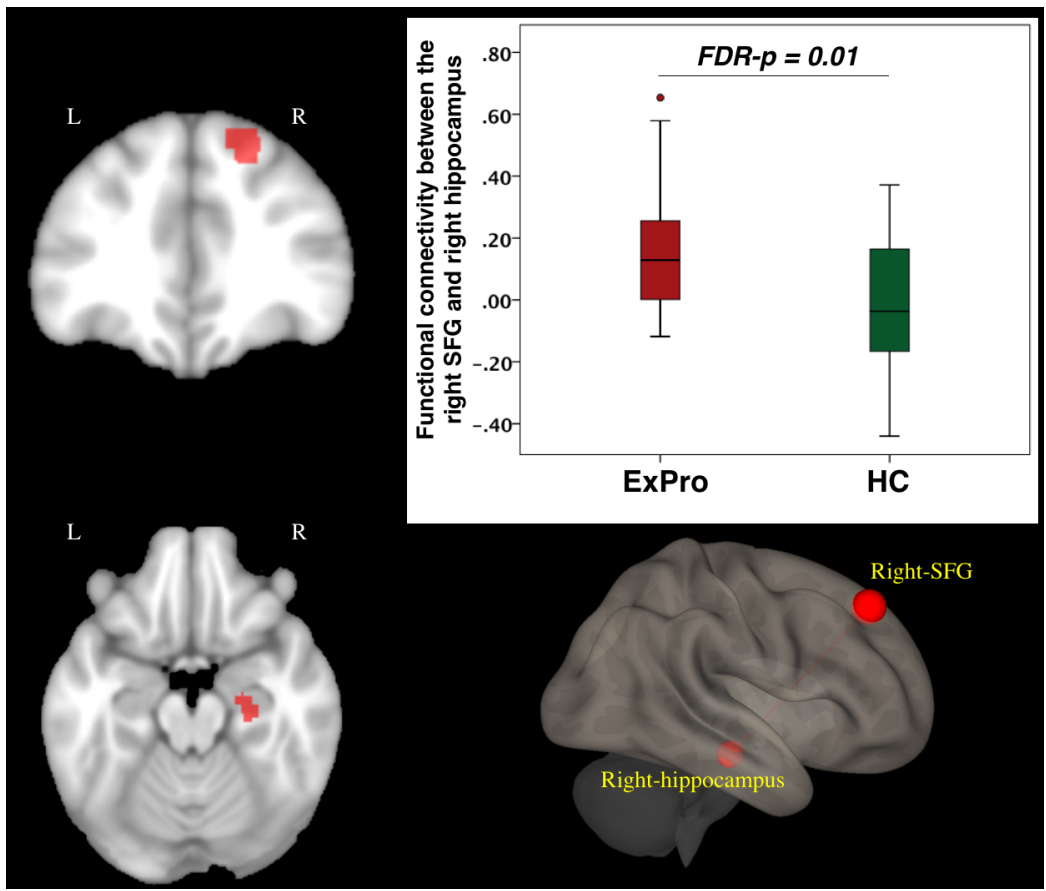

ene_14251_f1.jpeg 


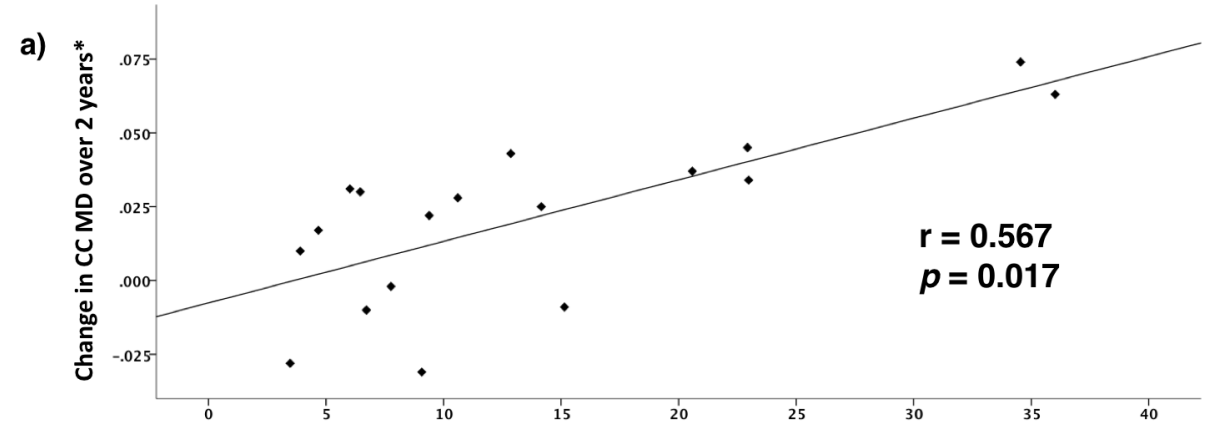

b)

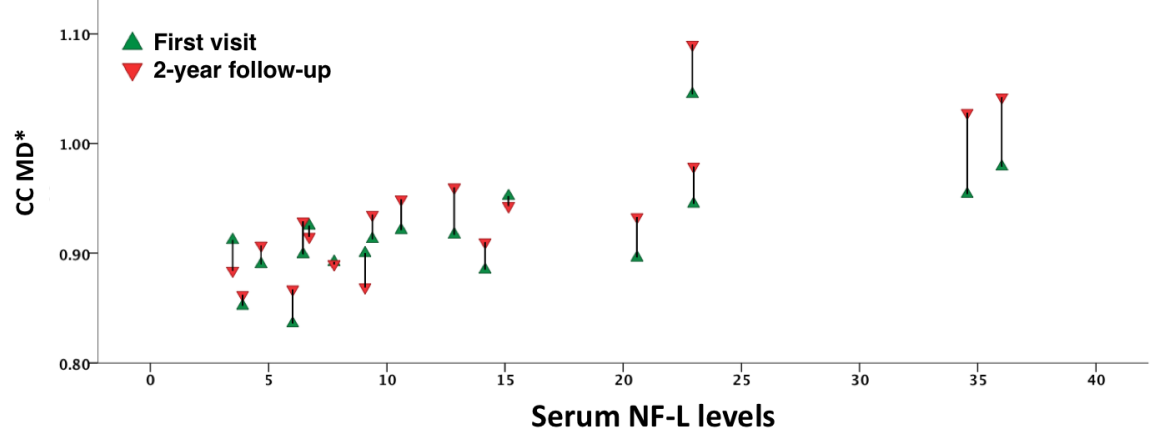

ene_14251_f2.jpeg 